\title{
LOCAL AND NON-LOCAL CONTRIBUTIONS TO HORIZONTAL MOTIONS IN STELLAR CONVECTIVE ZONES
}

\section{K. PETROVAY}

Eötvös University, Department of Astronomy, Budapest, Ludovika tér 2, H-1083 Hungary

ABSTRACT A method to determine the r.m.s. horizontal velocities in stellar convective zones (assuming that non-locality effects are not too strong) is given and applied to the solar case. The results may serve as useful input parameters for both convection theory and dynamo theory.

\section{METHOD}

Progress in stellar convection theory in the past decade has made it possible to break away from phenomenological models containing a large number of free parameters. The recent, more quantitative models (e.g. Unno and Kondo 1989, Canuto and Mazzitelli 1991) rely on the achievements of turbulence theory in their attempt to reduce the number of uncertain parameters. The velocity anisotropy of convection

$$
s(z):=\frac{\overline{v_{x}^{2}}(z)}{\overline{v_{z}^{2}}(z)}
$$

( $x$ and $y$ are horizontal coordinates, $\mathbf{v}$ is the velocity) is one such parameter to be specified. Here we present the results of a determination of the anisotropy as a function of depth in the solar convective zone on the basis of turbulence theory. (The effect of rotation will be neglected.)

Let us take the continuity equation (for simplicity in anelastic approximation and plane parallel geometry):

$$
v_{z} \frac{d \rho}{d z}+\rho \frac{\partial v_{z}}{\partial z}+\rho \frac{\partial v_{x}}{\partial x}+\rho \frac{\partial v_{y}}{\partial y}=0
$$

with $z$ the depth and $\rho$ the density.

Multiplying by $\rho v_{z}$ and averaging, we have

$$
\frac{1}{2} \frac{d}{d z}\left(\rho^{2} V_{z}^{2}\right)+2 \rho^{2} \overline{v_{z} \frac{\partial v_{x}}{\partial x}}=\frac{1}{2} \frac{d}{d z}\left(\rho^{2} V_{z}^{2}\right)-2 \rho^{2} \overline{v_{x} \frac{\partial v_{z}}{\partial x}}=0
$$

where the notation

$$
V_{i}=\left(\overline{v_{i}^{2}}\right)^{1 / 2}
$$

was introduced for the r.m.s. velocities. 
Now let us split $v_{x}$ (and $v_{y}$ ) into two (local + non-local) components:

$$
v_{x}=v_{x, l}+v_{x, n}
$$

so that

$$
\overline{v_{x, l} \frac{\partial v_{z}}{\partial x}}=0 \quad \text { while } \quad \overline{v_{x, n} \frac{\partial v_{z}}{\partial x}} \neq 0
$$

Now let us assume that $V_{x, l}^{2} \gg V_{x, n}^{2}$, i.e. that the non-locality effect is weak. In this case, supposing that $\rho(z), V_{z}^{2}(z)$ and $l(z)$ are known from a convective zone model, the local contributions $s_{l} \equiv V_{x, l}^{2} / V_{z}^{2}$ and $X \equiv l^{2} / l_{h}^{2}$ ( $l_{h}$ is the horizontal correlation length) can be computed from a homogeneous anisotropic turbulence model (Petrovay 1992). The non-local contribution $s_{n} \equiv V_{x, n}^{2} / V_{z}^{2}$ can be computed from equation (3):

$$
\begin{aligned}
& \frac{1}{2 \rho^{2}} \frac{d}{d z}\left(\rho^{2} V_{z}^{2}\right) \sim 2 V_{x, n} \frac{V_{z}}{l} X^{1 / 2} \\
& \Rightarrow \quad V_{x, n}=\frac{l}{2 \rho} X^{-1 / 2} \frac{d}{d z}\left(\rho V_{z}\right)
\end{aligned}
$$

and $s=s_{l}+s_{n}$ due to the orthogonality of the decomposition $\left(\overline{v_{x, l} v_{x, n}}=0\right)$.

If $\rho(z), V_{z}^{2}(z)$ and $l(z)$ are not a priori known, the above relations can simply be used together with the equations of the given convection theory to solve for all the unknown quantities.

Finally, using the above decomposition of the anisotropy the relations for the morphology of convection derived in Petrovay (1990) can more appropriately be written as

$$
f_{d}=\frac{1}{2}\left[1+\operatorname{sgn} F_{k i n}\left(\frac{A^{2}}{A^{2}+4}\right)^{1 / 2}\right]
$$

with

$$
A^{2}=\frac{F_{k i n}^{2}}{\rho^{2} V_{z}^{6}}\left(\frac{2}{2 s+1}\right)^{2}
$$

and

$$
f_{d, c r-l i m}=\operatorname{sgn} \frac{d\left(\rho V_{z}\right)}{d z} \min \left[\left(1+20 s_{n}\right)^{-1} ; 0.5\right]+0.5\left[1-\operatorname{sgn} \frac{d\left(\rho V_{z}\right)}{d z}\right] .
$$

Here, $f_{d}$ is the downflow filling factor (in a horizontal cross-section), $f_{d, c r-l i m}$ is the widest limit for the critical filling factor (topology reversal takes place in the regime $0.5>f_{d}>f_{d, c r-l i m}$ or $\left.0.5<f_{d}<f_{d, c r-l i m}\right)$, and $F_{k i n}$ is the kinetical energy flux.

\section{APPLICATION TO THE SOLAR CASE}

Our basic model will be the model $D$ in Unno and Kondo (1989). ( $l=H_{P}$ is supposed here). The functions $\rho(z), V_{z}^{2}(z)$ are taken from this model. This is somewhat inconsistent as the model is based on the assumption that $s \equiv 0.5$, 


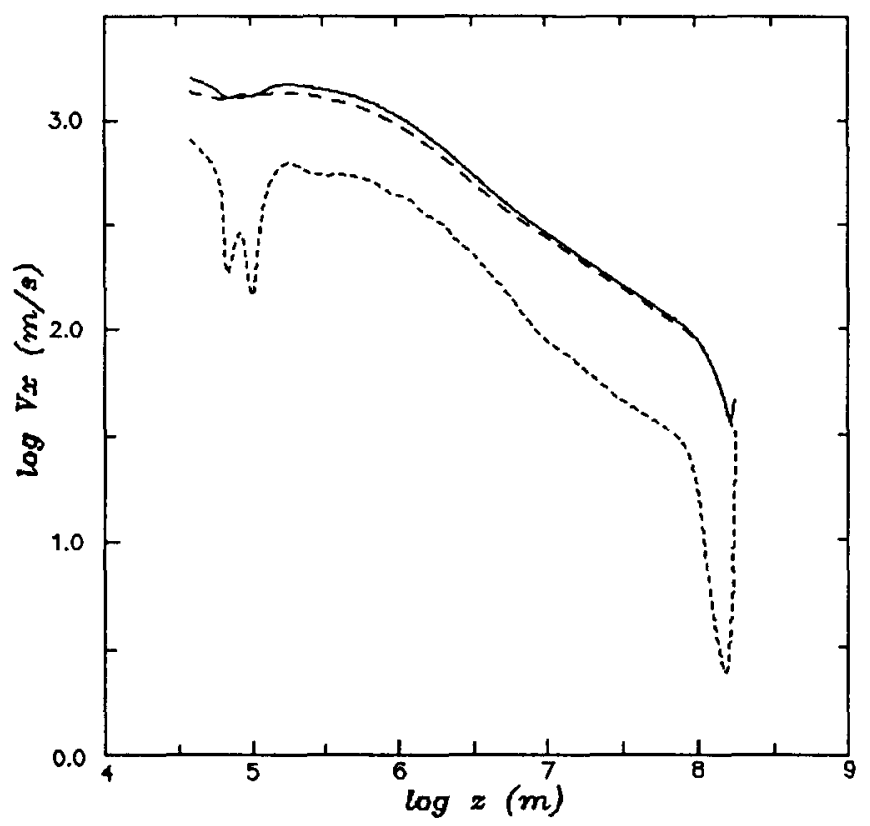

FIGURE I R.m.s. horizontal velocity as a function of depth in the solar convective zone. Solid: total r.m.s. velocity; long dashes: local contribution; short dashes: non-local contribution.

the results however show that this assumption is a posteriori confirmed with the exception of the neighbourhood of the boundaries of the solar convective zone. The two contributions to the horizontal velocities are shown on Figure I. Finaily, it must be noted again that the present approach breaks down near the borders of the convective zone where strongly non-local effects come into play.

\section{ACKNOWLEDGMENTS}

This work was funded by the OTKA (grant no. 2135) as well as by the Hungarian Science Foundation (grant no. 23/91/III) and Hungarian High Education Foundation (grant no. 69/91) of the Hungarian Credit Bank.

\section{REFERENCES}

Canuto, V. M. and Mazzitelli, I. 1991, Ap. J. 370, 295.

Petrovay, K. 1990, Ap. J. 362, 722.

Petrovay, K. 1992, Geophys. Astrophys. Fluid Dyn., in press

Unno, W. and Kondo, M. 1989, P.A.S. Japan 41, 197. 